Portland State University

PDXScholar

$1-1-1996$

\title{
Friction-Weighted Self-Consistent Effective Binary Diffusion Approximation
}

John D. Ramshaw

Portland State University, jdramshaw@yahoo.com

C. H. Chang

Idaho National Engineering Laboratory

Follow this and additional works at: https://pdxscholar.library.pdx.edu/phy_fac

Part of the Physics Commons

Let us know how access to this document benefits you.

Citation Details

J.D. Ramshaw and C.H. Chang, "Friction-Weighted Self-Consistent Effective Binary Diffusion Approximation," J. Non-Equilib. Thermodyn. 21, 223 (1996)

This Article is brought to you for free and open access. It has been accepted for inclusion in Physics Faculty Publications and Presentations by an authorized administrator of PDXScholar. Please contact us if we can make this document more accessible: pdxscholar@pdx.edu. 


\title{
Friction-Weighted Self-Consistent Effective Binary Diffusion Approximation*
}

\author{
J. D. Ramshaw, C. H. Chang \\ Idaho National Engineering Laboratory, Idaho Falls, U.S.A. \\ Communicated by J. V. Sengers, College Park, U.S.A. \\ Registration Number 696
}

\section{Abstract}

The self-consistent effective binary diffusion (SCEBD) approximation for multicomponent diffusion in gas mixtures is reconsidered and reformulated. The new formulation is based on the fact that a suitable rearrangement of the Stefan-Maxwell equations provides an exact expression for the complementary mean velocity $\mathbf{a}_{i}$ for species $i$ as a weighted average of the velocities of all the other species. The coefficients in $\mathbf{a}_{i}$ are normalized friction coefficients which are simply related to the true binary diffusion coefficients. A simple factorized bilinear approximation to the friction coefficients then yields approximate species diffusion fluxes identical in form to those of a previous intuitive treatment [4], together with a new relation between the previously ambiguous weighting factors $w_{i}$ and the friction coefficients. This relation places the SCEBD approximation on a firm foundation by providing a rational basis for determining the $w_{i}$. A simple further approximation based on the known form of the friction coefficients for hard spheres yields $w_{i}=$ (const.) $\rho_{i} / \sqrt{M_{i}}$, where $\rho_{i}$ and $M_{i}$ are respectively the mass density and molecular weight of species $i$. These weighting factors are shown to produce considerably more accurate diffusion velocities than the conventional choice $w_{i}=$ (const.) $\rho_{i} / M_{i}$.

\section{Introduction and summary}

A correct treatment of diffusion in multicomponent gas mixtures requires the solution of a linear system of equations for the species diffusion velocities or mass fluxes. These equations take the form [1]

$$
\sum_{j} \frac{x_{i} x_{j}}{D_{i j}}\left(\mathbf{u}_{j}-\mathbf{u}_{i}\right)=\mathbf{G}_{i}
$$

\footnotetext{
* Work performed under the auspices of the U. S. Department of Energy under DOE Field Office, Idaho Contract DE-AC07-94ID13223.
} 
where $x_{i}$ and $\mathbf{u}_{i}$ are respectively the mole fraction and specific velocity of species $i, D_{i j}$ is the binary diffusivity for the pair $(i, j)$, and the driving forces $\mathbf{G}_{i}$ are given by

$$
\mathbf{G}_{i}=\nabla x_{i}+\left(x_{i}-y_{i}\right) \nabla \ln p+K_{i} \nabla \ln T-\frac{1}{p}\left(\rho_{i} \mathbf{F}_{i}-y_{i} \sum_{j} \rho_{j} \mathbf{F}_{j}\right)
$$

where $y_{i}$ and $\rho_{i}$ are respectively the mass fraction and partial mass density of species $i$ $\left(y_{i}=\rho_{i} / \rho\right.$, where $\rho=\sum_{i} \rho_{i}$ is the total mass density), $p$ is the pressure, $T$ is the temperature, and $F_{i}$ is the body force per unit mass acting on species $i$. The coefficients $K_{i}$ are simply related to the species thermal diffusion coefficients [1], and have the property that $\sum_{i} K_{i}=0$. Equations (1) are frequently referred to as the Stefan-Maxwell equations [2]. They determine the $u_{i}$ to within a constant vector, the value of which is then determined by the relation

$$
\sum_{i} y_{i} \mathbf{u}_{i}=\mathbf{u}
$$

where $\mathbf{u}$ is the mass-averaged velocity of the mixture, which is determined by the mixture momentum equation. The diffusional mass flux of species $i$ relative to $\mathbf{u}$ is given by $\mathbf{J}_{i} \equiv \rho_{i}\left(\mathbf{u}_{i}-\mathbf{u}\right)$. It follows from equation (3) that $\sum_{i} \mathbf{J}_{i}=\mathbf{0}$, which may be regarded as a statement of mass conservation.

The solution of equations (1) presents an unwelcome and often costly complication in many situations, particularly in multidimensional numerical simulations where the diffusion fluxes $J_{i}$ are required at each mesh point and time step in the calculation. For this reason, effective binary diffusion approximations [2-4] are often used to avoid solving these equations. Several such approximations have been employed, most of which have suffered from the fundamental inconsistency that the condition $\sum_{i} \mathbf{J}_{i}=\mathbf{0}$ is violated. However, the origin of this inconsistency has recently been identified and a rational procedure for self-consistently removing it has been developed [4], so this is no longer a problem.

Unfortunately, such approximations have also suffered from a second serious problem which has received little previous attention. The problem concerns an ambiguity which was implicit in earlier work but manifested itself explicitly in the treatment of [4]. The ambiguity arises in the following manner. The basic idea of an effective binary diffusion approximation is to approximate $\mathbf{J}_{\boldsymbol{i}}$ by regarding the multicomponent mixture as a binary mixture of species $i$ and a complementary composite species representing all the other species. Since these species move with different velocities, the effective velocity $\mathbf{a}_{i}$ of the composite species should evidently be some appropriate weighted average of the velocities of the species it represents. However, the proper choice of the associated weighting factors $w_{i}$ is not obvious. (The traditional choice in most of the earlier work has been $w_{i}=x_{i}[2,3]$. This choice is exact in the trivial case when all the $D_{i j}$ are equal, but appears to have no fundamental basis otherwise.) The ambiguity in the choice of weighting factors then renders the entire approximation ambiguous, so that one obtains a whole family of self-consistent effective binary diffusion approximations rather than a single approximation. This situation is clearly unsatisfactory. 
Our purpose here is to reformulate the self-consistent effective binary diffusion (SCEBD) approximation [4] in such a way that this ambiguity does not arise:. The development is based on the fact that a suitable rearrangement of the Stefan-Maxwell equations provides an exact expression for the velocity $\mathbf{a}_{i}$ as a weighted average of the velocities $u_{j}$ with $j \neq i$. The coefficients in this expression are normalized friction coefficients which are simply related to the ordinary binary diffusion coefficients. A simple factorization approximation to the friction coefficients then leads to approximate $\mathbb{J}_{i}$ identical in form to those obtained in [4], together with a new relation between the previously ambiguous weighting factors $w_{i}$ and the friction coefficients or binary diffusion coefficients $D_{i j}$. The $w_{i}$ thereby acquire a new interpretation as parameters in a well-defined approximation to the friction coefficients. This places the SCEBD approximation on a new and firmer foundation by providing a rational basis for determining the $w_{i}$.

Unfortunately, the new relation between the weighting factors and friction coefficients cannot be solved for the $w_{i}$ in closed form. Further approximations are therefore required to obtain explicit expressions for the $w_{i}$. Suitable approximations for the case in which the number of species $N$ is large are presented in an appendix. These approximations make no assumptions as to the form of the friction coefficients, but in the present context this generality is rather more of a vice than a virtue. The friction coefficients are ordinarily obtained from kinetic theory, which provides their explicit dependence on molecular weights and other molecular parameters. The known form of these dependences can then be exploited to obtain a better approximation for the $w_{i}$. For general use, we therefore propose a simple approximation based on the known form of the friction coefficients for hard spheres. This approximation yields weighting factors of the very simple form $w_{i}=$ (const.) $\rho_{i} / \sqrt{M_{i}}$, where $M_{i}$ is the molecular weight of species $i$. We assess the accuracy of this choice by selecting several arbitrary mixtures of species of widely different molecular weights, randomly generating a large number of combinations of mole fractions and driving forces, and examining the residuals obtained by substituting the resulting approximate $\mathbf{u}_{i}$ into equation (1). These residuals are found to be considerably smaller than those obtained using either the conventional choice $w_{i}=x_{i}$ or the alternative simple intuitive choice $w_{i}=y_{i}$. We therefore conclude and recommend that the new weighting factors $w_{i}=$ (const.) $\rho_{i} / \sqrt{M_{i}}$ should supersede the conventional ones $w_{i}=x_{i}$ in future applications of the SCEBD approximation.

\section{Friction coefficients and weighting factors}

The binary diffusion coefficients $D_{i j}$ in equation (1) are inversely proportional to friction coefficients [5], which are frequently easier to work with. For present purposes, it is convenient to define friction coefficients $\alpha_{i j} \equiv x_{i} x_{j} / D_{i j}$, so that equation (1) becomes

$$
\sum_{j} \alpha_{i j}\left(\mathbf{u}_{j}-\mathbf{u}_{i}\right)=\mathbf{G}_{i}
$$

Since the $D_{i j}$ are symmetric in $(i, j)$, the same is true for the friction coefficients; i.e., $\alpha_{i j}=\alpha_{j i}$. The $\alpha_{i j}$ are fundamentally defined only for $i \neq j$. In order to avoid the 
restriction $i \neq j$ on subsequent summations, it is convenient to further define $\alpha_{i i} \equiv 0$ for all $i$. Equation (4) can then be rewritten in the form

$$
\mathbf{u}_{i}=-\mathbf{G}_{i} / \alpha_{i}+\mathbf{a}_{i}
$$

where

$$
\alpha_{i} \equiv \sum_{j} \alpha_{i j}=x_{i} \sum_{j \neq i} \frac{x_{j}}{D_{i j}}
$$

and

$$
\mathbf{a}_{i}=\sum_{j}\left(\alpha_{i j} / \alpha_{i}\right) \mathbf{u}_{j}
$$

No approximations have yet been made, and we observe that equation (7) rigorously expresses $\mathbf{a}_{i}$ as a linear combination or weighted average of the $\mathbf{u}_{j}$ for $j \neq i$. The latter interpretation follows from equation (6), which implies that $\sum_{j}\left(\alpha_{i j} / \alpha_{i}\right)=1$.

\section{Approximation of the friction coefficients}

We now proceed to seek an approximation' to $\mathbf{a}_{\boldsymbol{i}}$ which permits equation (5) to be explicitly solved for the $\mathbf{u}_{i}$. This will be done by means of a suitable approximation to the friction coefficients $\alpha_{i j}$ in equation (7). The simplest such approximation which satisfies both the condition $\alpha_{i i}=0$ and the symmetry condition $\alpha_{i j}=\alpha_{j i}$ is evidently a bilinear or factorization approximation of the form

$$
\alpha_{i j} \cong w_{i} w_{j}\left(1-\delta_{i j}\right)
$$

where $\delta_{i j}$ is the Kronecker delta and the $w_{i}$ are yet to be determined. There are $N(N-1) / 2$ independent nonzero $\alpha_{i j}$ to be approximated, and we have only $N$ parameters $w_{i}$ at our disposal, so equation (8) cannot in general be satisfied exactly for $N>3$. We merely aspire to satisfy it in some appropriate average sense, of which the obvious choice is consistency with the correct values of the $\alpha_{i}$. Thus we combine equations (6) and (8) to obtain

$$
\alpha_{i}=w_{i}\left(w-w_{i}\right)
$$

where $w \equiv \sum_{j} w_{j}$. The $w_{i}$ are now determined by equation (9).

Combining equations (6)-(8), we obtain

$$
\mathbf{a}_{i}=\left(w-w_{i}\right)^{-1} \sum_{j} w_{j}\left(1-\delta_{i j}\right) \mathbf{u}_{j}=\left(w-w_{i}\right)^{-1} \sum_{j \neq i} w_{j} \mathbf{u}_{j}
$$

which combines with equation (5) to yield

$$
\mathbf{u}_{i}=\mathbf{a}-\left(1-\frac{w_{i}}{w}\right) \frac{\mathbf{G}_{i}}{\alpha_{i}}
$$


where

$$
w \mathbf{a}=\sum_{j} w_{j} \mathbf{u}_{j}=w_{i} \mathbf{u}_{i}+\left(w-w_{i}\right) \mathbf{a}_{i} .
$$

Equation (11) may be rewritten in the form

$$
\mathbf{u}_{i}=\mathbf{a}-\left(D_{i} / x_{i}\right) \mathbf{G}_{i}
$$

where the effective binary diffusivities $D_{i}$ are defined by

$$
D_{i} \equiv\left(1-\frac{w_{i}}{w}\right) \frac{x_{i}}{\alpha_{i}}=\left(1-\frac{w_{i}}{w}\right)\left(\sum_{j \neq i} \frac{x_{j}}{D_{i j}}\right)^{-1} .
$$

Equations (11) and (12) may be regarded as a system of $N+1$ equations in the $N+1$ unknowns $\mathbf{u}_{i}$ and $\mathbf{a}$. However, only $N$ of these equations are linearly independent, as can be seen by combining equations (11) and (12) to obtain

$$
\sum_{i} w_{i}\left(1-\frac{w_{i}}{w}\right) \frac{\mathbf{G}_{i}}{\alpha_{i}}=\mathbf{0}
$$

which is identically satisfied by virtue of equations (2) and (9). It follows that equations (11) and (12) do not determine a, so that equation (11) or (13) determines the $\mathbf{u}_{i}$ only to within an additive constant vector. This is to say, equation (11) or (13) determines only differences between species velocities, just like the original StefanMaxwell equations (1) or (4). The additional relation needed to determine a and close the system is simply equation (3), which combines with equation (13) to yield

$$
\mathbf{a}=\mathbf{u}+\sum_{i}\left(y_{i} D_{i} / x_{i}\right) \mathbf{G}_{i}
$$

Equation (16) may now be used to eliminate a from equation (13), with the result

$$
\mathbf{u}_{i}=\mathbf{u}-\left(D_{i} / x_{i}\right) \mathbf{G}_{i}+\sum_{j}\left(y_{j} D_{j} / x_{j}\right) \mathbf{G}_{j}
$$

The corresponding self-consistent diffusion fluxes $\mathbf{J}_{i}=\rho_{i}\left(\mathbf{u}_{i}-\mathbf{u}\right)$ are then given by

$$
\mathbf{J}_{i}=-c M_{i} D_{i} \mathbf{G}_{i}+y_{i} c \sum_{j} M_{j} D_{j} \mathbf{G}_{j}
$$

where $c=\sum_{i} \rho_{i} / M_{i}$ is the total molar concentration.

It is noteworthy that, in contrast to previous effective binary diffusion approximations, the present approach does not produce an inconsistent set of diffusion velocities which require the correction procedure of Ref. [4] to restore consistency with equation (3). We obtain instead a consistent set of approximate diffusion velocities which are indeterminate to within a constant vector $\mathbf{a}$. This indeterminacy corresponds precisely 
to that of the original Stefan-Maxwell equations themselves, and it is satisfying that the present approximation has properly preserved this feature.

We now observe that equations (12)-(14) and (18) are identical in form to equations (13)-(16) of [4]. Thus the present development confirms the formal structure of the SCEBD approximation presented in [4], for which it provides a more cogent justification. In addition, the present formulation has the important and highly desirable new feature that the weighting factors $w_{i}$ are no longer arbitrary or ambiguous. Equation (9) removes this ambiguity by expressing the $w_{i}$ in terms of the friction coefficients $\alpha_{i j}$. We also note parenthetically that equations (9) and (14) combine to imply that $w_{i}$ is proportional to $x_{i} / D_{i}$, a relation which was previously suggested on intuitive grounds [4].

\section{Approximation of the weighting factors}

The weighting factors $w_{i}$ are determined by equation (9), which has the formal solution

$$
w_{i}=\frac{1}{2}\left(w-\sqrt{w^{2}-4 \alpha_{i}}\right)
$$

where the minus sign has been taken to ensure that both sides of the equation have the same sign in the special case when all the $\alpha_{i}$ are equal. However, equation (19) does not really provide an explicit solution for $w_{i}$ or $w_{i} / w$ because the condition $\sum_{i} w_{i}=w$ has not yet been enforced. The value of $w$ is implicitly determined by combining this condition with equation (19) to obtain

$$
\sum_{i} \sqrt{1-4 \alpha_{i} / w^{2}}=N-2
$$

This equation determines $w$, which then combines with equation (19) to determine the $w_{i}$. Equation (20) is unfortunately not amenable to an analytical solution for $w$. It could easily be solved numerically, but this would be contrary to the spirit of a simple approximation, and moreover could hardly be justified since equation (8) is only approximate in any case. It is therefore of interest to develop simple further approximations which lead to explicit expressions for the $w_{i} / w$.

Approximations to $w_{i} / w$ which are suitable for large $N$ and arbitrary $\alpha_{i j}$ are derived in the Appendix. Here, however, we shall propose an alternative simple approximation which exploits the fact that the $\alpha_{i j}$ are not simply arbitrary numbers but rather are known functions of the molecular weights and other molecular parameters. These functions are provided by the kinetic theory of gases $[1,5]$ and they are of course not of the simple form assumed in equation (8). By examining their true structure, however, we may hope to sensibly approximate them in that form. To this end, we consider the known form of $\alpha_{i j}$ for hard spheres, namely $[1,5]$

$$
\alpha_{i j}=A \frac{\rho_{i}}{\sqrt{M_{i}}} \frac{\rho_{j}}{\sqrt{M}_{j}} \frac{\sigma_{i j}}{{\sqrt{M_{i}+M_{j}}}}\left(1-\delta_{i j}\right)
$$

J. Non-Equilib. Thermodyn. Vol. 21, 1996, No. 3 
where $A$ is a constant independent of $(i, j)$, and $\sigma_{i j}$ is the cross-section for $(i, j)$ collisions. We now observe that if we neglect the relatively weak dependence of the factor $\sigma_{i j} / \sqrt{M_{i}+M_{j}}$ on $(i, j)$, we obtain $\alpha_{i j}$ in precisely the form of equation (8) with $w_{i}$ proportional to $\rho_{i} / \sqrt{M_{i}}$. This suggests the simple approximation

$$
\frac{w_{i}}{w} \cong \frac{\rho_{i} / \sqrt{M_{i}}}{\sum_{j} \rho_{j} / \sqrt{M_{j}}}
$$

for the normalized weighting factors required by equation (14). It is noteworthy that this weighting is just the normalized geometric mean of the conventional choice $w_{i} / w=x_{i}$ and the alternative intuitive choice $w_{i} / w=y_{i}$. Equation (22) should be superior to either of these choices, and this is confirmed in the next section.

\section{Accuracy}

In order to assess the accuracy of the new weighting factors given by equation (22) relative to the simple intuitive choices $w_{i} / w=x_{i}$ and $y_{i}$, we have conducted numerical tests for several arbitrary mixtures of the species shown in Table 1. The Lennard-Jones parameters $\sigma_{i}$ and $\varepsilon_{i}$ for these species were obtained from [6], and the diffusion coefficients $D_{i j}$ were computed using the formula $[1,6]$

$$
D_{i j}=D_{0} \frac{\left(T^{3} / M_{i j}\right)^{1 / 2}}{p \sigma_{i j}^{2} \Omega\left(k_{B} T / \sqrt{\varepsilon_{i} \varepsilon_{j}}\right)}
$$

where $D_{0}=1.8829 \times 10^{-13} \mathrm{~g}^{3 / 2} \mathrm{~cm}^{3} \mathrm{~s}^{-3} \mathrm{~mole}^{-1 / 2} K^{-3 / 2}, M_{i j}=M_{i} M_{j} /\left(M_{i}+M_{j}\right)$, $\sigma_{i j}=(1 / 2)\left(\sigma_{i}+\sigma_{j}\right), k_{B}$ is Boltzmann's constant, and

$$
\Omega(z)=4(1+2 z)^{-2}+z^{-0.145} .
$$

Table 1: Species used in accuracy studies

\begin{tabular}{lccc}
\hline Species & $M_{i}(\mathrm{~g} / \mathrm{mol})$ & $\varepsilon_{i} / k_{B}(\mathrm{~K})$ & $\sigma_{i}(\mathrm{~A})$ \\
\hline $\mathrm{N}_{2}$ & 28 & 97.53 & 3.621 \\
$\mathrm{~N}$ & 14 & 71.40 & 3.298 \\
$\mathrm{H}_{2}$ & 2 & 38.00 & 2.920 \\
$\mathrm{H}$ & 1 & 145.00 & 2.050 \\
$\mathrm{O}_{2}$ & 32 & 107.40 & 3.458 \\
$\mathrm{O}$ & 16 & 80.00 & 2.750 \\
$\mathrm{OH}$ & 17 & 80.00 & 2.750 \\
$\mathrm{H}_{2} \mathrm{O}$ & 18 & 809.10 & 2.641 \\
$\mathrm{NO}$ & 30 & 97.53 & 3.621 \\
$\mathrm{CO}$ & 28 & 98.10 & 3.650 \\
$\mathrm{CO}$ & 44 & 244.00 & 3.763 \\
\end{tabular}

J. Non-Equilib Thermodyn. Vol. 21, 1996, No. 3 
For simplicity we restricted attention to diffusion in one dimension, where the vectors $\mathbf{u}_{i}$ and $\mathbf{G}_{i}$ reduce to scalars $u_{i}$ and $G_{i}$. All tests were performed at $p=1 \mathrm{~atm}$ and $T=1500 \mathrm{~K}$.

We considered four different mixtures, which were obtained by taking the first $N$ species from Table 1 for $N=3,5,8$, and 11 . For each of these mixtures, we used a standard pseudo-random number generator to produce a large number of random combinations of mole fractions $x_{i}$ and driving forces $G_{i}$ uniformly distributed in the intervals $0<x_{i}<1$ and $-1<G_{i}<1$ subject to the constraints $\sum_{i} x_{i}=1$ and $\sum_{i} G_{i}=0$. Each complete set of the random $x_{i}$ and $G_{i}$ constitutes a single realization, and averages were taken over a total of $N_{r}$ realizations. The results were found to be insensitive to $N_{r}$ for $N_{r}>5000$, so the final data presented below were all obtained by setting $N_{r}=5000$.

For each realization $v$, we first evaluated the $u_{i}$ from equation (17). We then computed the species-averaged normalized Stefan-Maxwell residuals defined by

$$
R_{v}=\frac{1}{N} \sum_{i} \frac{\left|S_{i}-G_{i}\right|}{\max \left(\left|S_{i}\right|,\left|G_{i}\right|\right)}
$$

where $S_{i}$ denotes the left member of equation (1). Finally, these residuals were averaged over all realizations to obtain the overall mean residual $R=N_{r}^{-1} \sum_{v} R_{v}$. The resulting mean residuals $R$ are shown in Table 2 . We note that $R$ decreases monotonically with increasing $N$ for both $w_{i} / w=x_{i}$ and $y_{i}$, indicating that the SCEBD approximation has a general tendency to become more accurate for larger $N$ as one might intuitively expect. (It is also noteworthy that the conventional choice $w_{i} / w=x_{i}$ is generally less accurate than the choice $w_{i} / w=y_{i}$.) The new weighting factors of equation (22) exhibit the same behavior for $N>5$, but they also show a sharp reduction in $R$ for $N=3$. The main point of interest, however, is that equation (22) produces significantly more accurate results than either $w_{i} / w=x_{i}$ or $y_{i}$ in all four mixtures. The residuals resulting from these other choices are larger than those of equation (22) by as much as a factor of three for $N=3$, and by factors ranging from about 1.3 to 1.7 in the other cases. Moreover, the overall level of accuracy resulting from equation (22) is surprisingly good for such a simple approximation. The errors range from a high of $10 \%$ for $N=5$ down to $3 \%$ for $N=11$. In contrast, the choices $w_{i} / w=x_{i}$ and $y_{i}$ produce errors of nearly $20 \%$ for $N=3$, decreasing to about $5 \%$ for $N=11$. We therefore conclude that the new weighting

Table 2: Mean residual $R$ for different weighting factors

\begin{tabular}{lllll}
\hline & $\mathrm{N}=3$ & $\mathrm{~N}=5$ & $\mathrm{~N}=8$ & $\mathrm{~N}=11$ \\
\hline Eq. (16) & 0.0594 & 0.0965 & 0.0500 & 0.0345 \\
$w_{i} / w=x_{i}$ & 0.1951 & 0.1643 & 0.0851 & 0.0581 \\
$w_{i} / w=y_{i}$ & 0.1776 & 0.1429 & 0.0669 & 0.0440 \\
\hline
\end{tabular}


factors of equation (22) are markedly superior to either of the simple intuitive choices $w_{i} / w=x_{i}$ or $y_{i}$.

\section{Acknowledgments}

We are grateful to Dr. L. D. Cloutman for supplying us with Ref. [6] and for many helpful discussions.

\section{Appendix}

As discussed in the main text, we shall here attempt to determine the $w_{i}$ from equation (9) alone, without regard to the functional form or values of the $\alpha_{i j}$. Since $\sum_{i} w_{i}=w$, we would ordinarily expect that $w_{i} \sim w / N$, so that $w_{i} \ll w$ when $N$ is large. In this case, it should be a good approximation to neglect $w_{i}$ relative to $w$ in equation (9), with the result

$$
\alpha_{i} \cong w w_{i}
$$

Summing over $i$, we obtain $w^{2} \cong \alpha_{0}$, where $\alpha_{0} \equiv \sum_{i} \alpha_{i}=\sum_{i j} \alpha_{i j}$. It follows that

$$
w_{i} / w \cong \alpha_{i} / \alpha_{0}
$$

which should be reasonably accurate for large $N$. For smaller $N$, an improved approximation can be generated by considering equation (A2) as an initial estimate to be used in evaluating the factor $\left(w-w_{i}\right)$ in equation (9). This leads to the second-order approximation

$$
\frac{w_{i}}{w} \cong\left(\sum_{j} \frac{\alpha_{j}}{1-\alpha_{j} / \alpha_{0}}\right)^{-1} \frac{\alpha_{i}}{1-\alpha_{i} / \alpha_{0}}
$$

This procedure could be continued to higher orders if desired, but to go much further would again be contrary to the spirit of the enterprise.

Accuracy studies similar to those of Section 5 show that for $N \geq 4$, the use of equation (A2) or (A3) produces results of about the same accuracy as equation (22), with equation (A3) slightly better than equation (A2) as expected. For $N=3$, however, equation (22) gives considerably better results than equation (A2) or (A3), at least for the mixtures considered in Section 5.

\section{References}

[1] Hirschfelder, J. O., Curtiss, C. F., Bird, R. B., Molecular Theory of Gases and Liquids, Wiley, New York, 1954.

[2] Bird, R. B., Stewart, W. E., Lightfoot, E. N., Transport Phenomena, Wiley, New York, 1960.

[3] Williams, F. A., Combustion Theory, 2nd ed., Benjamin/Cummings, Menlo Park, CA, 1985.

[4] Ramshaw, J. D., Self-consistent effective binary diffusion in multicomponent gas mixtures, $J$. Non-Equilib. Thermodyn., 15 (1990), 295.

[5] Ramshaw, J. D., Hydrodynamic theory of multicomponent diffusion and thermal diffusion in multitemperature gas mixtures, J. Non-Equilib. Thermodyn., 18 (1993), 121. 
[6] Cloutman, L. D., A Database of Selected Transport Coefficients for Combustion Studies, Lawrence Livermore National Laboratory Report UCRL-ID-115050 (1993).

Paper received: 1995-6-28

Paper accepted: 1995-12-1

Dr. John D. Ramshaw

Lawrence Livermore National Laboratory

Dr. C. H. Chang

University of California

Idaho National Engineering Laboratory

P.O. Box 808, L-018

Livermore, CA 94550

Mail Stop 3808

P. O. Box 1625

U.S.A.

Idaho Falls, ID 83415

U.S.A. 